\title{
CYP2J2 participates in atherogenesis by mediating cell proliferation, migration and foam cell formation
}

\author{
RUI LI，YUAN ZHANG，HUACHENG YAN，HUA XIAO，YUNJUN RUAN，JIAN QIU and LEI SHI \\ Department of Cardiology, Guangzhou General Hospital of Guangzhou Military Command, \\ Guangzhou, Guangdong 510010, P.R. China
}

Received November 16, 2015; Accepted November 14, 2016

DOI: $10.3892 / \mathrm{mmr} .2016 .6039$

\begin{abstract}
Atherosclerosis (AS) is a common pathological basis for the development of various cardiovascular and cerebrovascular diseases, however, currently, no effective treatment against AS has been established. It has previously been suggested that intravascular cytochrome P450 (CYP) oxidase is involved in the pathogenesis of AS. The present study investigated the role of cytochrome P450, family 2, subfamily J, polypeptide 2 (CYP2J2), the most common subtype of CYP oxidase in the human body, in the occurrence and development of AS. CYP2J2 was overexpressed in human umbilical vein endothelial cells (HUVECs), human arterial smooth muscle cells (HASMCs), and human peripheral monocyte-derived foam cells by lentiviral infection. The mRNA and protein levels were measured by reverse-transcription quantitative polymerase chain reaction and western blotting, respectively. Cell proliferation and migration were determined by MTS and Transwell assays, respectively. Furthermore, lipid accumulation was detected with Oil red $\mathrm{O}$ staining. The concentrations of total and free cholesterol were measured using a quantitation kit. Following lentiviral infection, CYP2J2 was successfully overexpressed in HUVEC, HASMC and foam cells. CYP2J2 overexpression promoted proliferation and migration in HUVECs and suppressed these actions in HASMCs. In addition, it suppressed oxidized low-density lipoprotein-induced foam cell formation. In conclusion, it was hypothesized that CYP2J2 may have a protective role in AS, as proliferation of HASMCs and the formation of foam cells are notable characteristics of AS.
\end{abstract}

Correspondence to: Dr Jian Qiu or Dr Lei Shi, Department of Cardiology, Guangzhou General Hospital of Guangzhou Military Command, 111 Liuhua Road, Guangzhou, Guangdong 510010, P.R. China

E-mail: qiujian53568xn@163.com

E-mail: lucyshi622._921@163.com

Key words: atherosclerosis, cytochrome $\mathrm{P} 450$ family 2 subfamily $\mathrm{J}$ member 2, proliferation, migration, foam cell formation

\section{Introduction}

Atherosclerosis (AS) is commonly the pathological basis for numerous cardiovascular and cerebrovascular diseases. Worldwide, the mortality rate from cardiovascular diseases has been predicted to reach $36 \%$ by 2020 (1). AS develops progressively, however, an effective treatment against it is yet to be established. Given the increasing average human life expectancy and the aging population, AS poses a prominent threat to human health. Therefore, active research investigating the pathogenesis of AS and establishing effective therapeutic targets has become a critical issue.

Notable characteristics of AS include arterial intimal lipid deposition, proliferation of smooth muscle cells (SMCs) and connective tissues, infiltration and proliferation of monocytes/macrophages, and the formation of foam cells (which cause focal intimal fibrous thickening and plaque formation leading to hardening of the arteries and artery stenosis) (2). Multiple mechanisms have been proposed to be involved in AS development, including the thrombogenic, lipid infiltration, homocysteine, and smooth muscle mutation theories, and also the monoclonal, response-to-injury, oxidative, arginine, shear stress and stem cell hypotheses (3). However, none of these comprehensively explain the pathological development of AS. Numerous studies have suggested that intravascular cytochrome P450 (CYP) oxidase is involved in AS pathogenesis (4-12).

It has previously been demonstrated that overexpression of CYP oxidase has a protective effect on tumor necrosis factor $\alpha$-induced endothelial cell apoptosis (12). CYP oxidase promotes proliferation and migration of bovine aortic endothelial cells (9). In vivo, overexpression of CYP epoxygenase reduces blood pressure $(6,10)$, proinflammatory protein expression and low-density lipoprotein (LDL) cholesterol levels in the blood, however it increases high-density lipoprotein cholesterol (11). Inhibition of CYP oxidase causes contrasting effects (4). These results indicate that the CYP oxidase family of proteins is important in the regulation of cardiovascular diseases.

Based on this functional role of the CYP family, we hypothesized that genetic and environmental factors downregulate the expression of CYP oxidase, and lead to its dysfunction. The protective effect on the blood vessels is subsequently reduced or lost, leading to the development of AS. The present 
study used cytochrome P450 family 2 subfamily J member 2 (CYP2J2), the most common subtype of CYP oxidase in the human body, for the investigation. The effects of CYP2J2 overexpression on the proliferation and migration of human venous endothelial cells, arterial SMCs and human peripheral monocyte-derived foam cell formation were investigated. The current study presents a preliminary report on the effects of CYP2J2 on the occurrence and development of AS.

\section{Materials and methods}

Cell lines and culture. Human umbilical vein endothelial cells (HUVECs) were purchased from the China Center for Type Culture Collection (Wuhan, China). They were cultured in RPMI-1640 medium supplemented with $10 \%$ fetal bovine serum (FBS; Hyclone; GE Healthcare Life Sciences, Logan, UT, USA), penicillin $(100 \mathrm{U} / \mathrm{ml})$ and streptomycin $(100 \mu \mathrm{g} / \mathrm{ml})$. Primary cultured human aortic smooth muscle cells (HASMC) were purchased from ScienCell Research Laboratories (San Diego, CA, USA). These were maintained in Smooth Muscle Cell Medium (cat. no. 1101; ScienCell Research Laboratories). All cells were maintained at $37^{\circ} \mathrm{C}$ in a humidified incubator in an atmosphere containing $5 \% \mathrm{CO}_{2}$, and passaged when $90-95 \%$ confluent. Trypsin $(0.25 \%)$ was used for digestion and passaging.

Peripheral blood mononuclear cell (PBMC) isolation and foam cell culture. Fresh blood was anonymously collected from 5 coronary heart disease patients with approval from the Guangzhou General Hospital of Guangzhou Military Area Command of Chinese PLAq (Guangzhou, China), and all patients provided written informed consent for the use of samples for the present study. PBMCs were isolated by density-gradient centrifugation at $400 \mathrm{x} \mathrm{g}$ for $30 \mathrm{~min}$ at $20^{\circ} \mathrm{C}$ using Ficoll-Paque ${ }^{\mathrm{TM}}$ PLUS (GE Healthcare Life Sciences, Uppsala, Sweden). They were then seeded onto 96-well plates at $2 \times 10^{5}$ cells per well and cultured at $37^{\circ} \mathrm{C}$ with $5 \%$ $\mathrm{CO}_{2}$. The cells that adhered to the plate after $3 \mathrm{~h}$ were considered to be monocytes and were induced to differentiate into monocyte-derived macrophages for 2 days in RPMI-1640 medium containing $50 \mathrm{nM}$ phorbol-12-myristate-13-acetate. The medium was replenished each day. PBMC-derived macrophages were transformed into foam cells by incubation with oxidized LDL (ox-LDL; $80 \mathrm{mg} / \mathrm{l}$ ) for $48 \mathrm{~h}$, and subsequently infected with lentivirus (LV). After $72 \mathrm{~h}$, the culture medium and cells were harvested for total cholesterol measurement and Oil red O staining, respectively.

$L V$ packaging and cell infection. The complete open reading frame of CYP2J2 (NM_000775.2) was amplified by polymerase chain reaction (PCR). Primer sequences were as follows: Forward, 5'-CCGCTCGAGGCCACCATGCTCGCGGCG ATGGGCTC-3' and reverse, 5'-CGCGGATCCTTACACCTG AGGAACAGCGCAGAG-3', containing XhoI and BamHI restriction sites within the $5^{\prime}$ and $3^{\prime}$ termini, respectively. The amplicon was inserted into the pLVX-IRES-ZsGreen1 plasmid (Clontech Laboratories, Inc., Mountainview, CA, USA), which was digested with the same enzymes. pLVXs encoding CYP2J2 or empty vector were generated by transient transfection of 293 T cells with Lipofectamine 2000 reagent (Invitrogen;
Thermo Fisher Scientific, Inc., Waltham, MA, USA) following the manufacturer's protocol. Vector production, concentration, and titration were performed in accordance with general procedures as previously described $(13,14)$. For infection, $2 \times 10^{5}$ HUVECs and foam cells were subcultured in 6-well culture plates for $24 \mathrm{~h}$ prior to transduction. The cell culture media were then removed and cells washed twice with PBS followed by addition of $0.5 \mathrm{ml}$ lentiviral suspension $\left[1 \times 10^{8} \mathrm{IU} / \mathrm{ml}\right.$, multiplicity of infection $(\mathrm{MOI})=100]$ containing $8 \mu \mathrm{g} / \mathrm{ml}$ polybrene. The cells were incubated at $37^{\circ} \mathrm{C}$ overnight. The vector suspension was aspirated, and fresh growth medium ( $2 \mathrm{ml} /$ flask) was added to the transduced cells followed by incubation at $37^{\circ} \mathrm{C}$ with $5 \% \mathrm{CO}_{2}$. The medium was replaced after $24 \mathrm{~h}$ and transduction efficiencies were evaluated on day 3 post-infection. The percentage of green fluorescent protein (GFP)-positive cells was determined by calculating the number of GFP-positive and total cells in randomly selected microscopic fields under a fluorescent microscope (Leica Microsystems GmbH, Wetzlar, Germany). Samples with $>80 \%$ GFP-positive cells were used for subsequent assays.

Cell proliferation assay. Cell proliferation was measured using the CellTiter 96 AQueous One Solution Cell Proliferation Assay kit (MTS; Promega Corporation, Madison, WI, USA) according to the manufacturer's protocol. CYP2J2-overexpressed and empty LV-infected HUVEC cells $\left(1 \times 10^{4}\right)$ were seeded onto 96 -well plates. Following culturing for $0,24,48$, and $72 \mathrm{~h}, 10 \mu \mathrm{l}$ CellTiter 96 AQueous One Solution reagent was added to each well and further incubated for $4 \mathrm{~h}$ at $37^{\circ} \mathrm{C}$. Absorbance at a wavelength of $490 \mathrm{~nm}$ was measured using a Multiskan MK3 microplate reader (Thermo Fisher Scientific, Inc.). The proliferation ratio was calculated using the following formula: Proliferation rate $(\%)=($ mean absorbance of LV-CYP2J2-GFP/mean absorbance of LV-GFP) x100.

$R N A$ extraction and reverse transcription-quantitative PCR (RT-qPCR) analysis. Total RNA was extracted from the harvested cells using TRIzol ${ }^{\circledR}$ reagent (Invitrogen; Thermo Fisher Scientific, Inc.). To quantify the mRNA levels of CYP2J2, RT was performed using GoScript Reverse Transcription System (Promega Corporation) according to the manufacturer's protocol, followed by qPCR using SYBR-Green qPCR SuperMix (Invitrogen; Thermo Fisher Scientific Inc.) on the ABI Prism ${ }^{\circledR} 7500$ Sequence Detection System (Applied Biosystems; Thermo Fisher Scientific, Inc.) The PCR cycling program involved an initial denaturation step at $95^{\circ} \mathrm{C}$ for $2 \mathrm{~min}$, followed by 40 cycles of $15 \mathrm{sec}$ at $95^{\circ} \mathrm{C}$ and $32 \mathrm{sec}$ at $60^{\circ} \mathrm{C}$. The present study used $18 \mathrm{~s}$ rRNA as an endogenous control. The primer sequences used were as follows: CYP2J2, forward, 5'-AGCACCCTGGACCTCTTCTT-3' and reverse, 5'-GGCCAATCACTCTGTCAATCT-3'; 18s rRNA, forward, 5'-CCTGGATACCGCAGCTAGGA-3' and reverse, 5'-GCG GCGCAATACGAATGCCCC-3'. Gene expression was measured in triplicates, quantified by the $2^{-\Delta \Delta C q}$ method (15), and normalized to a control.

Western blotting. Cells were lysed using radioimmunoprecipitation assay buffer (Beyotime Institute of Biotechnology, Haimen, China). Total protein concentration was determined using a bicinchoninic protein assay kit (Beyotime Institute 
of Biotechnology). Total protein $(30 \mu \mathrm{g})$ were separated by $10 \%$ SDS-PAGE and transferred onto polyvinylidene fluoride membranes (EMD Millipore, Billerica, MA, USA). The membranes were then blocked with $5 \%$ milk in Tris-buffered saline containing $0.05 \%$ Tween-20 (TBST) for $1 \mathrm{~h}$ at $37^{\circ} \mathrm{C}$, incubated for $1 \mathrm{~h}$ with anti-CYP2J2 mouse monoclonal primary antibody (1:500; cat. no. CF503636, OriGene Technologies, Inc., Beijing, China), washed three times with TBST, and further incubated with rabbit anti-mouse IgG $(\mathrm{H}+\mathrm{L})$-horseradish peroxidase (HRP) conjugated secondary antibody (1:5,000; cat. no. 6170-05, SouthernBiotech, Birmingham, AL, USA) for $40 \mathrm{~min}$. Subsequently, the membranes were washed three times with TBST. Membranes were blocked in TBST for $1 \mathrm{~h}$ at $37^{\circ} \mathrm{C}$ for GAPDH detection and incubated for $1 \mathrm{~h}$ with HRP-conjugated monoclonal mouse anti-GAPDH primary antibody (1:10,000; cat. no. KC-5G5, KangChen Biotech, Shanghai, China), and washed three times with TBST. All proteins were visualized using an enhanced chemiluminescence reagent (EMD Millipore).

Transwell migration. Cell migration and invasion were assessed using a Transwell assay. HUVECs and HASMCs were harvested, and $5 \times 10^{4}$ cells in $200 \mu$ 1 RPMI-1640 medium supplemented with $0.1 \%$ FBS were placed in the upper chamber of an insert (pore size, $8 \mu \mathrm{m}$ ). The lower chamber was filled with medium containing 10\% FBS $(600 \mu \mathrm{l})$. After $24 \mathrm{~h}$ of incubation and removal of cells on the upper chamber of the filter with a cotton swab, the cells on the underside were fixed with $4 \%$ paraformaldehyde for $20 \mathrm{~min}$ at $25^{\circ} \mathrm{C}$, stained with $0.1 \%$ crystal violet in $20 \%$ ethanol for $10 \mathrm{~min}$, and counted in five randomly selected fields using a phase contrast microscope. Migrating cells were monitored by imaging at x200 magnification under a light microscope (Olympus Corporation, Tokyo, Japan) in five independent fields for each well. The assays were performed in triplicate.

Oil red O staining of lipid accumulation in cells. Culture media was removed from PBMCs and cells were washed twice with PBS. The cells were then fixed with $4 \%$ paraformaldehyde for $30 \mathrm{~min}$. The Oil Red O working solution was prepared fresh from its $0.5 \%(\mathrm{w} / \mathrm{v})$ stock solution, which was diluted with water at a ratio of 6:4 (Oil Red O:water). Cells were then incubated with Oil Red $\mathrm{O}$ for $30 \mathrm{~min}$ at room temperature, washed gently with PBS three times to remove excess non-specific staining and observed under a light microscope (Olympus Corporation).

Quantitation of cellular cholesterol. Quantification of cellular total and free cholesterol was performed with culture media using the Total Cholesterol Quantitation kit (cat. no. F002-1) from Nanjing Jiancheng Bioengineering Institute (Nanjing, China) and Free Cholesterol Quantitation kit (cat. no. E1016) from Applygen Technologies, Inc. (Beijing, China), respectively. The detection was carried out according to the manufacturer's protocol. All measurements were conducted in duplicate, and all samples were analyzed in the same microplate to minimize run-to-run variability.

Statistical analyses. All data are presented as mean \pm standard deviation and analyzed using SPSS software version 19.0
(IBM SPSS, Armonk, NY, USA). One-way analysis of variance followed by a least significant difference post hoc test was used to determine statistical significance. $\mathrm{P}<0.05$ was considered to indicate a statistically significant difference.

\section{Results}

Overexpression of CYP2J2 in HUVEC, HASMC and foam cells. In order to understand the function of CYP2J2, LVs expressing CYP2J2 and GFP (LV-CYP2J2-GFP) or GFP alone (LV-GFP) were packaged and transduced into HUVECs, HASMCs and foam cells at MOI of 100. LV-CYP2J2-GFP and LV-GFP successfully infected all three cell types, as presented in Fig. 1A-C. The cells were harvested $72 \mathrm{~h}$ post-infection for RT-qPCR and western blotting analysis of CYP2J2 expression. CYP2J2 mRNA was significantly overexpressed in HUVECs $(\mathrm{P}=0.004)$, HASMCs $(\mathrm{P}<0.001)$ and foam cells $(\mathrm{P}<0.001)$, as detected by RT-qPCR (Fig. 1D-F). CYP2J2 protein levels were successfully overexpressed in HUVECs, HASMCs and foam cells, as detected western blotting (Fig. 1G-I).

CYP2J2 overexpression promotes proliferation of HUVECs and suppresses proliferation of HASMCs. To elucidate the possible function of CYP2J2 in atherosclerosis, the present study evaluated the effect off CYP2J2 on HUVEC and HASMC proliferation. Following $12 \mathrm{~h}$ of culture, CYP2J2 overexpression did not significantly affect HUVEC and HASMC proliferation compared with the LV-GFP-transduced cells $(\mathrm{P}=0.079)$. However, after 24,48 and $72 \mathrm{~h}$ of culture, CYP2J2 overexpression increased HUVEC proliferation by $10.0,19.6$ and $21.3 \%$, respectively, compared with the LV-GFP cells (Fig. 2A). However, HASMC proliferation was decreased by and 9,18 and $21 \%$ at 24,48 and $72 \mathrm{~h}$, respectively, compared with the LV-GFP cells (Fig. 2B).

Overexpression of CYP2J2 promotes migration of HUVECs and inhibits migration of HASMCs. The effect of CYP2J2 overexpression on HUVEC and HASMC migration was subsequently evaluated. The number of HUVECs that passed through the membrane into the lower chamber was significantly greater for LV-CYP2J2-GFP cells compared with LV-GFP cells $(\mathrm{P}=0.002)$. The number of migrating cells upon infection with LV-GFP and LV-CYP2J2-GFP was $58 \pm 14$ and $157 \pm 35$, respectively (Fig. 3A and B). The number of LV-CYP2J2-GFP HASMCs that passed through the membrane into the lower chamber was significantly reduced compared with LV-GFP cells $(\mathrm{P}=0.006)$. The number of migrating cells was $51 \pm 9$ and $16 \pm 5$ upon infection with LV-GFP and LV-CYP2J2-GFP, respectively (Fig. 3C and D).

CYP2J2 overexpression suppresses ox- $L D L$-induced foam cell formation. It has previously been demonstrated that cholesterol is involved in the pathogenesis of atherosclerosis, therefore the present study investigated the effect of CYP2J2 overexpression on lipid accumulation. PBMC-derived macrophages were treated with ox-LDL for $48 \mathrm{~h}$, and subsequently infected with LV-GFP or LV-CYP2J2-GFP. Following $72 \mathrm{~h}$ of infection, culture media were harvested for total cholesterol detection, while the cells were harvested for Oil Red O staining. Oil Red O staining was used to determine 
A

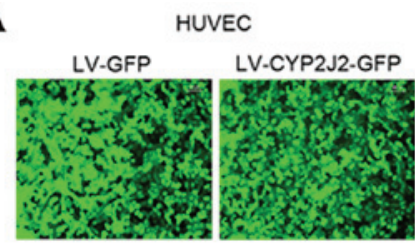

D

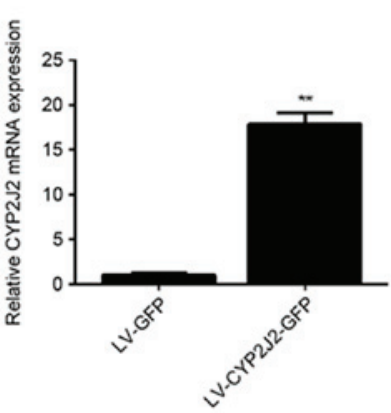

G

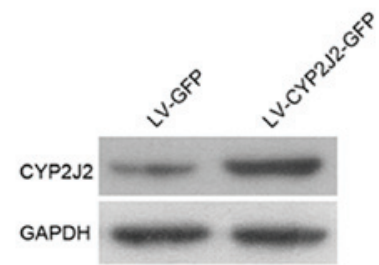

B

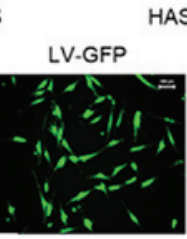

E

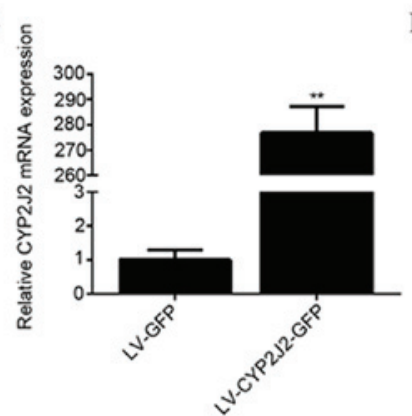

H

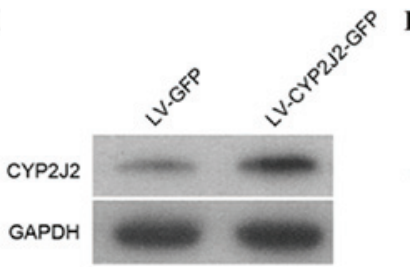

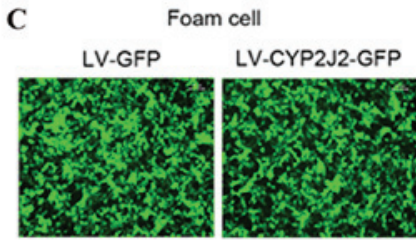

F
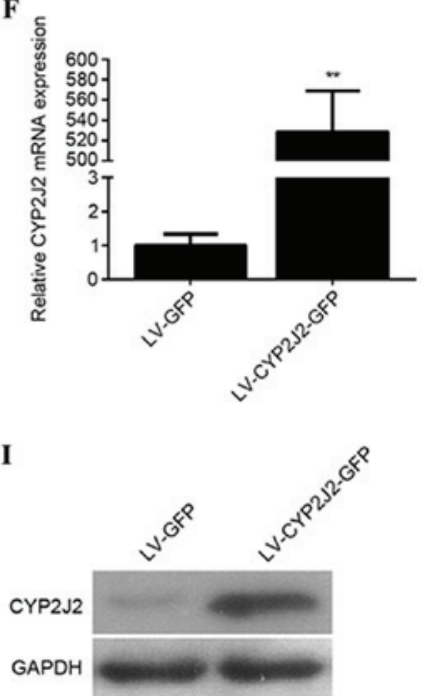

Figure 1. CYP2J2 is successfully overexpressed in HUVECs, HASMCs, and foam cells infected with LV-CYP2J2. (A) HUVEC, (B) HASMC and (C) foam cells were successfully infected with LV-CYP2J2-GFP or LV-GFP at MOI=100. Reverse transcription-quantitative polymerase chain reaction revealed that CYP2J2 was overexpressed in (D) HUVECs, (E) HASMCs and (F) foam cells. Western blot analysis verified that CYP2J2 was overexpressed in (G) HUVECs, (H) HAMSCs and (I) foam cells. CYP2J2, cytochrome P450 family 2 subfamily J polypeptide 2; HUVECs, human umbilical vein endothelial cells; HASMCs, human arterial smooth muscle cells; LV, lentivirus; GFP, green fluorescent protein. ${ }^{* *} \mathrm{P}<0.01 \mathrm{vs.} \mathrm{LV-GFP} \mathrm{group.}$

changes in cellular lipid accumulation following infection with LV-GFP or LV-CYP2J2-GFP. The results demonstrated that CYP2J2 overexpression markedly reduced lipid accumulation in foam cells (Fig. 4A). Furthermore, quantification of cellular cholesterol content revealed that total cholesterol $(\mathrm{P}=0.008)$ and free cholesterol $(\mathrm{P}=0.007)$ concentrations in LV-CYP2J2-GFP-infected cells were significantly lower compared with the LV-GFP-infected cells (Fig. 4B and C). CYP2J2 overexpression therefore effectively suppresses cellular cholesterol accumulation.

\section{Discussion}

The CYP oxidase family of proteins and its metabolites are important in the regulation of the development of cardiovascular diseases. CYP2J2 is the most common subtype of CYP oxidase in the human body and its function has been investigated in various cardiovascular diseases, including ischemic stroke, myocardial infarction, and coronary heart disease (16-18). Endothelial-specific CYP2J2 overexpression alleviates high-fat diet-induced hyperlipidemia in mice (19). In addition, CYP2J2 prevents cardiac fibrosis by suppressing the transmission of proinflammatory signals from cardiomyocytes to macrophages (20). CYP2J2 overexpression protects against susceptibility to arrhythmia in cardiac hypertrophy (21). Furthermore, polymorphism in cytochrome P450 epoxygenase $C Y P 2 J 2$ is associated coronary artery disease risk $(22,23)$. Therefore, based on the importance of CYP2J2 in cardiovascular diseases, the present study hypothesized that

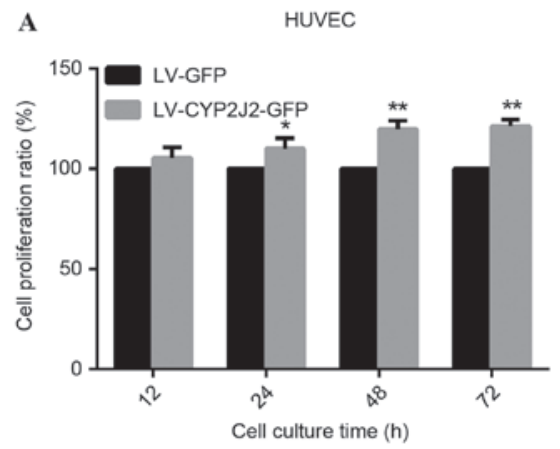

B HASMC

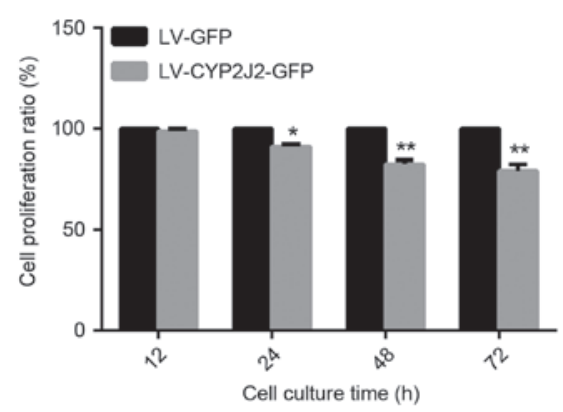

Figure 2. CYP2J2 overexpression promotes HUVEC and suppresses HASMC proliferation. Following infection for $72 \mathrm{~h}$ with LV-CYP2J2-GFP or LV-GFP, cells were harvested for cell proliferation assays. Absorbance at a wavelength of $490 \mathrm{~nm}$ was measured at 12, 24, 48, and $72 \mathrm{~h}$ time-points for (A) HUVECs and (B) HASMCs. Data are expressed as the mean \pm standard deviation. "P $<0.05$, ** $\mathrm{P}<0.01$ vs. LV-GFP. CYP2J2, cytochrome P450 family 2 subfamily J polypeptide 2; HUVECs, human umbilical vein endothelial cells; HASMCs, human arterial smooth muscle cells; LV, lentivirus; GFP, green fluorescent protein. 
A

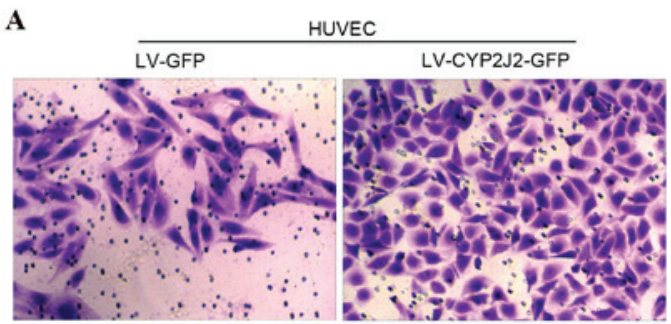

C

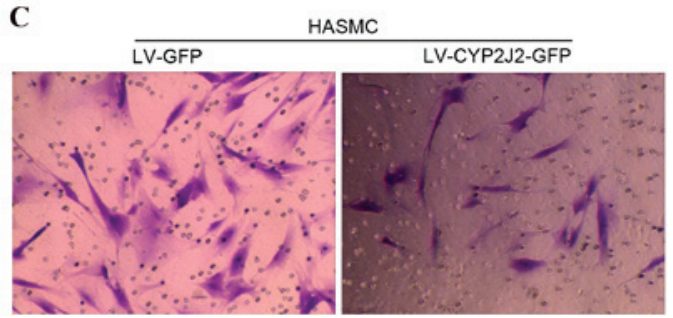

B

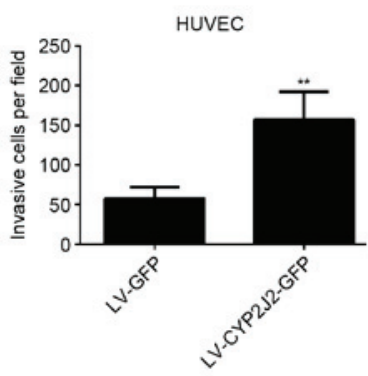

D

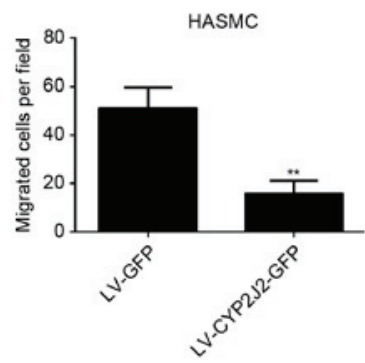

Figure 3. CYP2J2 overexpression promotes HUVEC migration and inhibits HASMC migration. (A) Representative images of crystal violet-stained HUVECs (magnification, x200) and (B) average numbers of migrating HUVECs per field for the experimental groups. (C) Representative images of crystal violet-stained HASMC (magnification, x200) and (D) average numbers of migrating HAMSCs per field for the experimental groups. The data are presented as the mean \pm standard deviation. ${ }^{* *} \mathrm{P}<0.01$ vs. LV-GFP. CYP2J2, cytochrome P450 family 2 subfamily J polypeptide 2; HUVECs, human umbilical vein endothelial cells; HASMCs, human arterial smooth muscle cells; LV, lentivirus; GFP, green fluorescent protein.
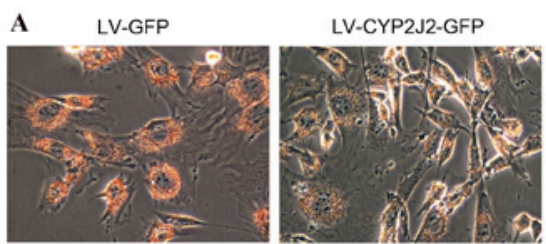

B
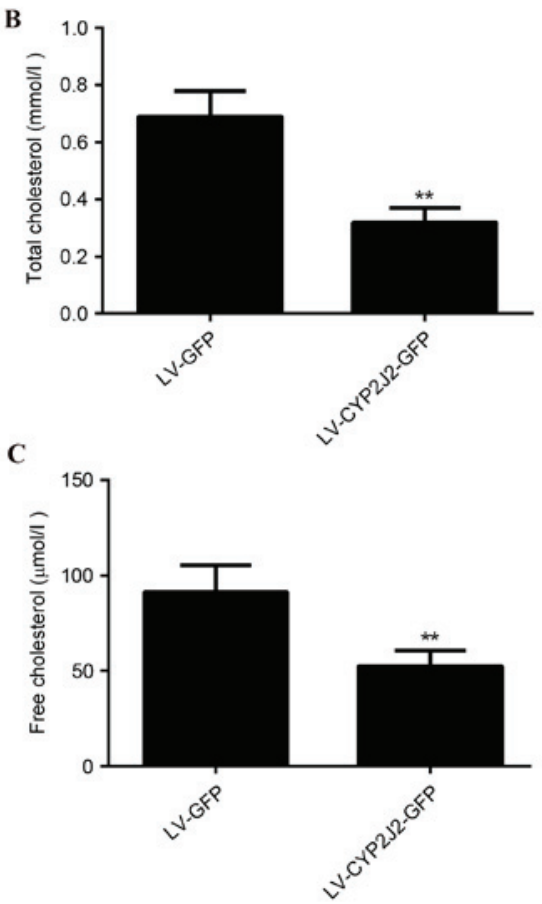

Figure 4. CYP2J2 overexpression suppresses oxidized low density lipoprotein-induced foam cell formation. (A) Oil Red O staining was used to detect alterations in cellular lipid accumulation following infection with LV-GFP or LV-CYP2J2-GFP. Magnification x200. (B) Total and (C) free cholesterol in culture medium were determined using a quantitation kit. Data are presented as the mean \pm standard deviation. ${ }^{* *} \mathrm{P}<0.01$ vs. LV-GFP. CYP2J2, cytochrome P450 family 2 subfamily J polypeptide 2; LV, lentivirus; GFP, green fluorescent protein.
CYP2J2 may regulate the occurrence and development of AS. AS is initiated by complex interactions between circulating factors and various cell types in the vessel walls, including endothelial cells, lymphocytes, monocytes, and SMCs (24). Therefore, the function of CYP2J2 in AS was investigated by observing the effects of its overexpression on the proliferation and migration of HUVECs, HASMCs and human peripheral monocyte-derived foam cell formation. The results provide a mechanistic basis and a novel therapeutic target for AS.

Endothelial cells are central to the cardiovascular system by regulating blood circulation and fluidity, vascular tone, coagulation, inflammatory responses and angiogenesis (9). Proliferation and migration of endothelial cells is an important process during angiogenesis and vessel sprouting. The response-to-injury hypothesis, suggests that endothelial cell injury is a factor in the occurrence and development of AS (25). It is therefore important to protect and recover the function of endothelial cells in AS prevention and therapy. The present study observed that CYP2J2 overexpression promoted proliferation and migration of HUVECs. This is supported by a previous study conducted by Wang et al (9), where the authors demonstrated that CYP oxidase promotes the proliferation and migration of bovine aortic endothelial cells (9). CYP2J2 may therefore exhibit a protective role in the endothelial cell injury process and promote angiogenesis and vessel sprouting. In addition, proliferation and migration of vascular smooth muscle cells (VSMCs), which are the major cell type in blood vessel walls, have been suggested to foster atherosclerosis development $(26,27)$. Furthermore, CYP2J2 overexpression suppressed HASMC proliferation and migration. This observation supports the suggestion that CYP2J2 suppresses VSMC hyperplasia and migration in the process of AS and protects against it. AS is a chronic disease characterized by the deposition of excessive cholesterol in the arterial intima (2). Uncontrolled uptake of ox-LDL, excessive 
cholesterol esterification, and/or impaired cholesterol release leads to accumulation of cholesterol ester (CE). CE is stored as cytoplasmic lipid droplets and subsequently triggers the formation of foam cells (28). The results of the present study revealed that CYP2J2 overexpression suppressed ox-LDL-induced foam cell formation. Formation of macrophage foam cells in the intima is a major hallmark of early-stage atherosclerotic lesions. Therefore, the aforementioned results further support the hypothesis that CYP2J2 exhibits a protective function against AS.

In conclusion, the present study demonstrated that overexpression of CYP2J2 promoted an angiogenic phenotype, which included endothelial cell proliferation and migration. CYP2J2 overexpression suppressed HASMC proliferation and migration, and ox-LDL-induced foam cell-formation processes that are a requisite for the occurrence and development of AS. Therefore, CYP2J2 may exhibit a protective role in the development of AS.

\section{Acknowledgements}

The present study was supported by Natural Science Foundation of Guangdong Province, China (grant no. S2013010011957) and National Natural Science Foundation of China (grant no. 81402671).

\section{References}

1. Negi S and Anand A: Atherosclerotic coronary heart disease-epidemiology, classification and management. Cardiovasc Hematol Disord Drug Targets 10: 257-261, 2010.

2. Weber C and Noels H: Atherosclerosis: Current pathogenesis and therapeutic options. Nat Med 17: 1410-1422, 2011.

3. Chen $\mathrm{Z}$ and $\mathrm{Xu} \mathrm{H}$ : Anti-inflammatory and immunomodulatory mechanism of tanshinone IIA for atherosclerosis. Evid Based Complement Alternat Med 2014: 267976, 2014.

4. Athirakul K, Bradbury JA, Graves JP, DeGraff LM, Ma J, Zhao Y, Couse JF, Quigley R, Harder DR, Zhao X, et al: Increased blood pressure in mice lacking cytochrome P450 2J5. FASEB J 22: 4096-4108, 2008

5. Bodiga S, Zhang R, Jacobs DE, Larsen BT, Tampo A, Manthati VL, Kwok WM, Zeldin DC, Falck JR, Gutterman DD, et al: Protective actions of epoxyeicosatrienoic acid: Dual targeting of cardiovascular PI3K and KATP channels. J Mol Cell Cardiol 46: 978-988, 2009.

6. Lee CR, Imig JD, Edin ML, Foley J, DeGraff LM, Bradbury JA, Graves JP, Lih FB, Clark J, Myers P, et al: Endothelial expression of human cytochrome P450 epoxygenases lowers blood pressure and attenuates hypertension-induced renal injury in mice. FASEB J 24: 3770-3781, 2010.

7. Node K, Huo Y, Ruan X, Yang B, Spiecker M, Ley K, Zeldin DC and Liao JK: Anti-inflammatory properties of cytochrome P450 epoxygenase-derived eicosanoids. Science 285: 1276-1279, 1999.

8. Node K, Ruan XL, Dai J, Yang SX, Graham L, Zeldin DC and Liao JK: Activation of Galpha s mediates induction of tissue-type plasminogen activator gene transcription by epoxyeicosatrienoic acids. J Biol Chem 276: 15983-15989, 2001.

9. Wang Y, Wei X, Xiao X,Hui R, Card JW, Carey MA, Wang DW and Zeldin DC: Arachidonic acid epoxygenase metabolites stimulate endothelial cell growth and angiogenesis via mitogen-activated protein kinase and phosphatidylinositol 3-kinase/Akt signaling pathways. J Pharmacol Exp Ther 314: 522-532, 2005.
10. Xiao B, Li X, Yan J, Yu X, Yang G, Xiao X, Voltz JW, Zeldin DC and Wang DW: Overexpression of cytochrome P450 epoxygenases prevents development of hypertension in spontaneously hypertensive rats by enhancing atrial natriuretic peptide. J Pharmacol Exp Ther 334: 784-794, 2010.

11. Xu X, Zhang XA and Wang DW: The roles of CYP450 epoxygenases and metabolites, epoxyeicosatrienoic acids, in cardiovascular and malignant diseases. Adv Drug Deliv Rev 63: 597-609, 2011.

12. Yang S, Lin L, Chen JX, Lee CR, Seubert JM, Wang Y, Wang H, Chao ZR, Tao DD, Gong JP, et al: Cytochrome P-450 epoxygenases protect endothelial cells from apoptosis induced by tumor necrosis factor-alpha via MAPK and PI3K/Akt signaling pathways. Am J Physiol Heart Circ Physiol 293: H142-H151, 2007.

13. Zufferey R, Dull T, Mandel RJ, Bukovsky A, Quiroz D, Naldini L and Trono D: Self-inactivating lentivirus vector for safe and efficient in vivo gene delivery. J Virol 72: 9873-9880, 1998.

14. Dull T, Zufferey R, Kelly M, Mandel RJ, Nguyen M, Trono D and Naldini L: A third-generation lentivirus vector with a conditional packaging system. J Virol 72: 8463-8471, 1998.

15. Livak KJ and Schmittgen TD: Analysis of relative gene expression data using real-time quantitative PCR and the 2(-Delta Delta C(T)) Method. Methods 25: 402-408, 2001

16. Li Q, Zhao JH, Ma PJ, Su LL, Tao SB and Ji SB: Association of CYP2J2 gene polymorphisms with ischemic stroke. Int J Clin Exp Med 8: 8163-8167, 2015.

17. Arun Kumar AS, Kumar SS, Umamaheswaran G, Kesavan R, Balachandar J and Adithan C: Association of CYP2C8, CYP2C9 and CYP2J2 gene polymorphisms with myocardial infarction in South Indian population. Pharmacol Rep 67: 97-101, 2015.

18. Lee CR, North KE, Bray MS, Couper DJ, Heiss G and Zeldin DC: CYP2J2 and CYP2C8 polymorphisms and coronary heart disease risk: The atherosclerosis risk in communities (ARIC) study. Pharmacogenet Genomics 17: 349-358, 2007.

19. Zhang S, Chen G, Li N, Dai M, Chen C, Wang P, Tang H, Hoopes SL, Zeldin DC, Wang DW and Xu X: CYP2J2 overexpression ameliorates hyperlipidemia via increased fatty acid oxidation mediated by the AMPK pathway. Obesity (Silver Spring) 23: 1401-1413, 2015.

20. Yang L, Ni L, Duan Q, Wang X, Chen C, Chen S, Chaugai S, Zeldin DC, Tang JR and Wang DW: CYP epoxygenase 2J2 prevents cardiac fibrosis by suppression of transmission of pro-inflammation from cardiomyocytes to macrophages. Prostaglandins Other Lipid Mediat 116-117: 64-75, 2015.

21. Westphal C, Spallek B, Konkel A, Marko L, Qadri F, DeGraff LM, Schubert C, Bradbury JA, Regitz-Zagrosek V, Falck JR, et al: CYP2J2 overexpression protects against arrhythmia susceptibility in cardiac hypertrophy. PLoS One 8: e73490, 2013

22. Spiecker M, Darius H, Hankeln T, Soufi M, Sattler AM, Schaefer JR, Node K, Börgel J, Mügge A, Lindpaintner K, et al: Risk of coronary artery disease associated with polymorphism of the cytochrome P450 epoxygenase CYP2J2. Circulation 110: 2132-2136, 2004

23. Zhu Q, Fu Z, Ma Y, Yang H, Huang D, Xie X, Liu F, Zheng Y and Cha E: A novel polymorphism of the CYP2J2 gene is associated with coronary artery disease in Uygur population in China. Clin Biochem 46: 1047-1054, 2013.

24. Doran AC, Meller N and McNamara CA: Role of smooth muscle cells in the initiation and early progression of atherosclerosis. Arterioscler Thromb Vasc Biol 28: 812-819, 2008.

25. Ross R: The pathogenesis of atherosclerosis: A perspective for the 1990s. Nature 362: 801-809, 1993.

26. Lu Q, Qiu TQ and Yang H: Ligustilide inhibits vascular smooth muscle cells proliferation. Eur J Pharmacol 542: 136-140, 2006.

27. Eun SY, Ko YS, Park SW, Chang KC and Kim HJ: IL-1 $\beta$ enhances vascular smooth muscle cell proliferation and migration via P2Y2 receptor-mediated RAGE expression and HMGB1 release. Vascul Pharmacol 72: 108-117, 2015.

28. Yu XH, Fu YC, Zhang DW, Yin K and Tang CK: Foam cells in atherosclerosis. Clin Chim Acta 424: 245-252, 2013. 